\title{
Optimal systolic blood pressure in noncritically ill patients with acute kidney injury: A retrospective cohort study
}

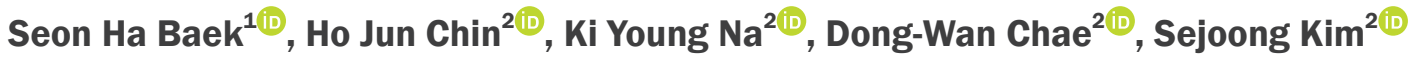 \\ 'Department of Internal Medicine, Hallym University Dongtan Sacred Heart Hospital, Hwaseong, Korea \\ ²Department of Internal Medicine, Seoul National University Bundang Hospital, Seongnam, Korea
}

\begin{abstract}
Background: Few data showed the optimal blood pressure (BP) in noncritically ill patients with acute kidney injury (AKI) relative to mortality or severe $\mathrm{AKI}$. We therefore sought to analyze the data that exist for the ideal target range for $\mathrm{BP}$ in noncritically ill patients with AKI.

Methods: We performed a retrospective cohort study involving 1,612 hospitalized patients who were diagnosed with AKI using the Kidney Disease: Improving Global Outcomes definition based on serum creatinine measurements for a period of 1 year. The average systolic BP (SBP) was categorized into 10-mmHg increments (within 48 hours after the development of AKI). The primary outcome was a composite of severe AKI or 90 -day mortality.

Results: The composite outcome rate in patients was $18.7 \%(302 / 1,612)$. The relationship between BP and the composite outcome followed a U-shaped curve, with an increased event rate observed at both low and high BP values. The average SBP after AKI predicted the composite outcome after adjusting for baseline variables (reference SBP: $120-129$ mmHg; < $100 \mathrm{mmHg}$ : hazard ratio [HR] 1.84, $P=0.015 ; 100-109 \mathrm{mmHg}$ : HR 1.56, $P=0.038$; 110-119 mmHg: HR 1.15, $P=0.483 ; 130-139$ mmHg: HR 1.51, $P=0.045 ; \geq 140$ mmHg: HR 1.73, $P=0.005$ ). Conclusion: Among noncritically ill patients with AKI, a U-shaped curve association was observed between the average SBP within 48 hours after AKI and the composite primary outcome of this study, with the lowest event rate for SBP ranging from approximately 110 to $129 \mathrm{mmHg}$.
\end{abstract}

Keywords: Acute kidney injury, Mortality, Noncritically ill patient, Systolic blood pressure

Received March 19, 2019; Revised June 17, 2019;

Accepted June 18, 2019

Edited by Dong-Ryeol Ryu, Ewha Womans University, Seoul, Korea Correspondence: Sejoong Kim

Department of Internal Medicine, Seoul National University Bundang Hospital, 82 Gumi-ro 173beon-gil, Bundang-gu, Seongnam 13620, Korea.E-mail:sejoong2@snu.ac.kr

Copyright (C) 2019 by The Korean Society of Nephrology

( ) This is an open-access article distributed under the terms of the Creative Commons Attribution Non-Commercial License (http://creativecommons. org/licenses/by-nc-nd/4.0/), which permits unrestricted non-commercial use, distribution, and reproduction in any medium, provided the original work is properly cited.

\section{Introduction}

Acute kidney injury (AKI) is a frequent and challenging medical complication with a high risk for morbidity and mortality [1]. The Kidney Disease: Improving Global Outcomes (KDIGO) group recommended that careful attention be paid to hemodynamic status during the treatment of patients with AKI [2] because blood pressure (BP) has been thought to be essential for organ perfusion [3].

Regarding the general management of hypertension, a recent randomized controlled trial (Systolic Blood Pressure Intervention Trial [SPRINT]) showed that lowering the systolic BP (SBP) to a target goal of $<120 \mathrm{mmHg}$ resulted in lower rates of fatal and nonfatal major cardio- 
vascular events and death [4]. This trial further demonstrated that adverse outcomes progressively and linearly increase with a rise in BP. Subsequently, a BP of $<120$ $\mathrm{mmHg}$ has been adopted as optimal in hypertensive patients. However, some data on acute illnesses such as acute coronary syndromes and stroke have suggested too low of a BP may be harmful [5-8]. In other words, a Jor U-shaped curve relationship existed between BP and mortality/cardiovascular outcomes.

Most studies considering hemodynamic targets in patients with AKI have been performed in critically ill patients [9-16]. Especially in patients with septic AKI or hepatorenal syndrome, a target mean arterial pressure (MAP) of 65 to $70 \mathrm{mmHg}$ has been suggested, while a higher target MAP of 80 to $85 \mathrm{mmHg}$ is recommended in patients with chronic hypertension [17].

However, few data cover the subject of optimal BP in noncritically ill patients with AKI relative to mortality or severe AKI, particularly when compared with critically ill patients. We thus sought to analyze the data that do exist to determine the best target range for $\mathrm{BP}$ in noncritically ill patients with AKI.

\section{Methods}

\section{Study population}

We collected data from 21,572 hospitalized patients aged 18 years or older whose serum creatinine (Cr) levels were identified once or more during their first admission for a period of 1 year (2013) at a tertiary care hospital in Seongnam, Korea. All admissions through general practice, accident, and emergency were included. Among them, 21,172 patients were deemed eligible for this study after excluding those with missing BP data $(\mathrm{n}=91)$, preexisting end-stage renal disease $(n=309)$, and intensive care unit admission during hospitalization $(n=3,048)$. Finally, we enrolled only 1,612 patients diagnosed with AKI according to the KDIGO definition based on serum Cr measurements (Fig. 1). All clinical investigations were conducted with respect to the 2008 Declaration of Helsinki and good clinical practice guidelines. This study was approved by the Institutional Review Board (IRB) of Seoul National University Bundang Hospital (IRB number: H-1508-310-115), with no written consent required because patient records/information were anonymized

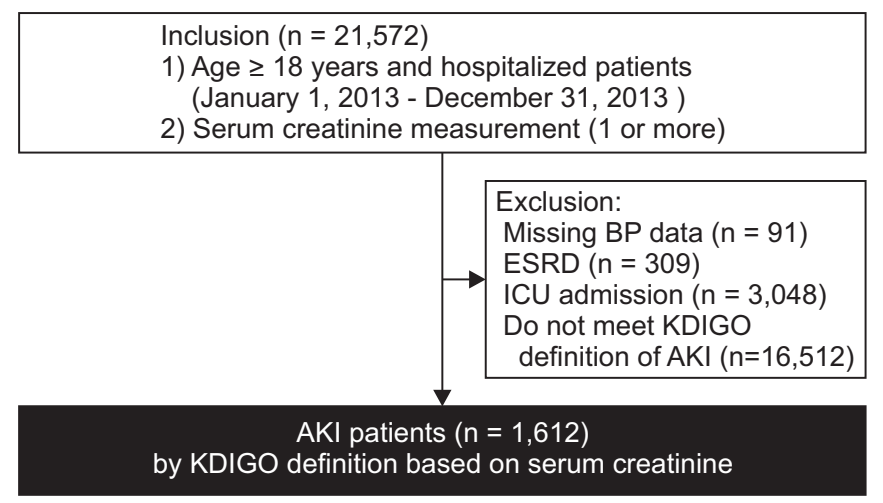

Figure 1. Algorithm for eligible patient selection.

AKI, acute kidney injury; BP, blood pressure; ESRD, end-stage renal disease; ICU, intensive care unit; KDIGO, Kidney Disease: Improving Global Outcomes.

and de-identified prior to analysis.

\section{Measurements and definitions}

Demographic, physiological, and laboratory data at the index admission were collected from an electronic medical records database. Serum $\mathrm{Cr}$ values were measured via the rate-blanked compensated kinetic alkaline picrate Jaffe kinetic method using an automatic analyzer (Toshiba-200FR; Toshiba Corp., Tokyo, Japan). The estimated glomerular filtration rate (eGFR) was calculated using the Modification of Diet in Renal Disease (MDRD) study equation [18] adjusted for a Korean ethnic coefficient [19]. To detect AKI, at least two Cr measurements were required: First taken during the baseline time window and a second recorded on a different day during hospital admission. We defined the baseline Cr level using the 6-month Cr data from before patients' admission, as this directly affected the detection and staging of AKI, in the manner as follows: By selecting the lowest $\mathrm{Cr}$ value measured less than 90 days prior to admission. If this value was unavailable, the lowest $\mathrm{Cr}$ value measured between 90 and 180 days before admission was considered. If this value was also unavailable, the $\mathrm{Cr}$ level was estimated using the MDRD study equation [18] adjusted for a Korean ethnic coefficient [19], assuming that the baseline eGFR was $75 \mathrm{~mL} / \mathrm{min} / 1.73 \mathrm{~m}^{2}$ [20]. Inpatient $\mathrm{Cr}$ measurements were defined as those obtained during admission. Using both a baseline and an inpatient $\mathrm{Cr}$ value measured on different days, AKI was defined as an increase of $0.3 \mathrm{mg}$ / $\mathrm{dL}$ or more in or of 1.5 times or more of the serum Cr, us- 
ing the serum $\mathrm{Cr}$ criteria proposed by the KDIGO [20]. Severe AKI was defined as stage III of the KDIGO using the serum $\mathrm{Cr}$ criteria (threefold increase in the serum $\mathrm{Cr}$ from baseline or increase in serum $\mathrm{Cr}$ to $4.0 \mathrm{mg} / \mathrm{dL}$ or more). We defined AKI solely based on changes in the measured serum $\mathrm{Cr}$ values because urine output data were not consistently available for all inpatients. Bacteremia was elucidated via positive blood culture during hospitalization. BacT/Alert FA and FN bottles (bio-Mérieux, Durham, NC, USA) were used for all blood cultures.

BP measurements were conducted using an electronic sphygmomanometer at the discretion of the nurse during the hospitalization period. For the present analysis, the average SBP was calculated for each patient within 48 hours after the development of AKI. The risk of mortality or severe AKI was then evaluated as a function of BP. BP values were categorized into $10-\mathrm{mmHg}$ increments to determine the association with mortality or severe AKI. Because the average follow-up BP variables represented the effects of BP over a period of time rather than at a single point in time, we considered this to be more important in predicting the outcomes.

We assessed the comorbidities based on the code of the International Classification of Disease, 10th Revision (ICD-10) as follows: cardiovascular disease including angina pectoris (I20), myocardial infarction (I21-I23), ischemic heart disease (I24-I25), and heart failure (I50); cancer (C); cerebrovascular disease (I63-I66); hypertension (I10-I15); and diabetes mellitus (E10-14). The presence of hypertension and diabetes mellitus were also confirmed by the use of antihypertensive medications and antihyperglycemic agents, respectively. Antihypertensive drugs included alpha- and beta-blockers, calcium-channel blockers, renin-angiotensin system inhibitors, and diuretics.

\section{Study outcome}

The composite outcome of 90-day all-cause mortality or severe AKI was considered in this research. We combined 90-day data from the Ministry of Security and Public Administration with our dataset using each individual's unique identifier. Mortality data were obtained until August 31, 2015.

\section{Statistical analysis}

Continuous variables were expressed as means \pm standard deviations, while categorical variables were expressed as percentages. Differences in continuous variables were analyzed using one-way analysis of variance or the Kruskal-Wallis rank test, whereas chi-square tests and

Table 1. Baseline characteristics of patients with AKI

\begin{tabular}{|c|c|}
\hline Characteristic & Total $(n=1,612)$ \\
\hline \multicolumn{2}{|l|}{ Average blood pressure (mmHg) } \\
\hline SBP within 48 hours after admission & $125.8 \pm 16.2$ \\
\hline DBP within 48 hours after admission & $69.9 \pm 10.1$ \\
\hline $\begin{array}{l}\text { SBP within } 48 \text { hours after the development } \\
\text { of AKI }\end{array}$ & $123.8 \pm 16.3$ \\
\hline $\begin{array}{l}\text { DBP within } 48 \text { hours after the development } \\
\text { of AKI }\end{array}$ & $68.3 \pm 10.4$ \\
\hline \multicolumn{2}{|l|}{ Demographics } \\
\hline Sex, male & $893(55.4)$ \\
\hline Age (yr) & $65.8 \pm 16.0$ \\
\hline Body mass index $\left(\mathrm{kg} / \mathrm{m}^{2}\right)$ & $23.3 \pm 3.9$ \\
\hline \multicolumn{2}{|l|}{ Comorbidity } \\
\hline Charlson's comorbidity index & $2.8 \pm 2.2$ \\
\hline Hypertension & $485(30.1)$ \\
\hline Diabetes mellitus & $574(35.6)$ \\
\hline \multicolumn{2}{|l|}{ Medication } \\
\hline RAAS blockade & $222(13.8)$ \\
\hline Beta-blocker & $143(8.9)$ \\
\hline Calcium-channel blocker & $198(12.3)$ \\
\hline Diuretics & $224(13.9)$ \\
\hline Alpha-blocker & $24(1.5)$ \\
\hline \multicolumn{2}{|l|}{ Laboratory values } \\
\hline Creatinine at baseline (mg/dL) & $1.00 \pm 1.09$ \\
\hline Creatinine at admission (mg/dL) & $1.57 \pm 1.72$ \\
\hline $\operatorname{WBC}\left(10^{9} / \mathrm{L}\right)$ & $10.0 \pm 16.9$ \\
\hline Hemoglobin (g/L) & $114.8 \pm 23.0$ \\
\hline Albumin $(\mathrm{g} / \mathrm{L})$ & $35.9 \pm 6.3$ \\
\hline Glucose (mmol/L) & $8.0 \pm 4.1$ \\
\hline Bilirubin, total $(\mu \mathrm{mol} / \mathrm{L})$ & $21.6 \pm 53.2$ \\
\hline Cholesterol, total (mmol/L) & $4.1 \pm 1.4$ \\
\hline $\mathrm{Na}(\mathrm{mmol} / \mathrm{L})$ & $137.5 \pm 4.8$ \\
\hline $\mathrm{K}(\mathrm{mmol} / \mathrm{L})$ & $4.2 \pm 0.7$ \\
\hline Total $\mathrm{CO}_{2}(\mathrm{mmol} / \mathrm{L})$ & $22.9 \pm 3.8$ \\
\hline Operation & $467(29.0)$ \\
\hline Sepsis & $133(8.3)$ \\
\hline
\end{tabular}

Data are presented as mean \pm standard deviation or number (\%). AKI, acute kidney injury; DBP, diastolic blood pressure; RAAS, reninangiotensin-aldosterone system; SBP, systolic blood pressure; WBC, white blood cell. 
Fisher's exact test were used for categorical variables. Cox's hazard proportion analysis was used to estimate the hazard ratios (HRs) for composite outcome according to the average SBP. We conducted the tests of proportional hazards assumptions and restricted cubic spline curves, respectively. The HRs and $95 \%$ confidence intervals (CIs) for the composite outcomes were calculated after a stepwise adjustment for multiple confounders including age, gender, body mass index, Charlson's comorbidity index, hypertension, operation, bacteremia, white blood cell count, hemoglobin, albumin, cholesterol, alanine aminotransferase, sodium, total $\mathrm{CO}_{2}$, and maximal $\mathrm{Cr}$ during admission.
The adjusted HR for each category of SBP was calculated based on the BP group in which the event rate was lowest, for which the HR was considered as $1 . P$ values of less than 0.05 were considered to be statistically significant. All analyses and calculations were conducted using the IBM SPSS Statistics version 24.0 (IBM Corp., Armonk, NY, USA) and STATA version 14.0 (StataCorp LP, College Station, TX, USA) software programs.

Table 2. Demographic baseline characteristics by average SBP categories after AKI

\begin{tabular}{|c|c|c|c|c|c|c|c|}
\hline & \multicolumn{6}{|c|}{ Mean SBP (mmHg) } & \multirow[b]{2}{*}{$P$ value } \\
\hline & $\begin{array}{c}<100 \\
(n=85)\end{array}$ & $\begin{array}{l}100-109 \\
(n=222)\end{array}$ & $\begin{array}{l}110-119 \\
(n=400)\end{array}$ & $\begin{array}{l}120-129 \\
(n=391)\end{array}$ & $\begin{array}{l}130-139 \\
(n=253)\end{array}$ & $\begin{array}{c}\geq 140 \\
(n=261)\end{array}$ & \\
\hline \multicolumn{8}{|l|}{ Demographics } \\
\hline Sex, male & $37(43.5)$ & $108(48.6)$ & $229(57.3)$ & $234(59.8)$ & $128(50.6)$ & $157(60.2)$ & 0.396 \\
\hline Age (yr) & $62.8 \pm 16.6$ & $63.8 \pm 15.9$ & $64.3 \pm 16.1$ & $66.4 \pm 16.7$ & $68.3 \pm 14.3$ & $67.3 \pm 15.8$ & 0.002 \\
\hline Body mass index $\left(\mathrm{kg} / \mathrm{m}^{2}\right)$ & $21.1 \pm 4.4$ & $22.8 \pm 3.7$ & $23.1 \pm 3.8$ & $23.4 \pm 4.0$ & $23.5 \pm 3.9$ & $24.3 \pm 4.1$ & $<0.001$ \\
\hline \multicolumn{8}{|l|}{ Comorbidity } \\
\hline Charlson's comorbidity index & $3.0 \pm 2.3$ & $3.1 \pm 2.3$ & $2.8 \pm 2.3$ & $2.6 \pm 2.1$ & $2.8 \pm 2.3$ & $2.6 \pm 2.3$ & 0.114 \\
\hline Hypertension & $21(24.7)$ & $58(26.1)$ & $106(26.5)$ & $120(30.7)$ & $68(26.9)$ & $112(42.9)$ & $<0.001$ \\
\hline Diabetes mellitus & $26(30.6)$ & $81(36.5)$ & $128(32.0)$ & $117(29.9)$ & 99 (39.1) & $123(47.1)$ & 0.001 \\
\hline \multicolumn{8}{|l|}{ Medication } \\
\hline RAAS blockade & $10(11.8)$ & $27(12.2)$ & $51(12.8)$ & $58(14.8)$ & $35(13.8)$ & $41(15.7)$ & 0.803 \\
\hline Beta-blocker & $3(3.5)$ & $15(6.8)$ & $30(7.5)$ & $38(9.7)$ & $24(9.5)$ & $33(12.6)$ & 0.069 \\
\hline Calcium-channel blocker & $4(4.7)$ & $20(9.0)$ & $39(9.8)$ & $46(11.8)$ & $27(10.7)$ & $62(23.8)$ & $<0.001$ \\
\hline Diuretics & $13(15.3)$ & $30(13.5)$ & $49(12.3)$ & $57(14.6)$ & $31(12.3)$ & $44(16.9)$ & 0.592 \\
\hline Alpha-blocker & $0(0)$ & $3(1.4)$ & $3(0.8)$ & $3(0.8)$ & $6(2.4)$ & $9(3.4)$ & 0.030 \\
\hline \multicolumn{8}{|l|}{ Laboratory values } \\
\hline Creatinine at baseline $(\mathrm{mg} / \mathrm{dL})$ & $0.86 \pm 1.29$ & $0.82 \pm 0.62$ & $0.87 \pm 0.56$ & $0.93 \pm 0.76$ & $1.11 \pm 1.44$ & $1.41 \pm 1.68$ & $<0.001$ \\
\hline Creatinine at admission (mg/dL) & $1.39 \pm 2.04$ & $1.18 \pm 0.89$ & $1.29 \pm 1.02$ & $1.38 \pm 1.14$ & $1.63 \pm 1.74$ & $2.64 \pm 2.89$ & $<0.001$ \\
\hline $\operatorname{WBC}\left(10^{9} / \mathrm{L}\right)$ & $11.1 \pm 12.3$ & $10.1 \pm 9.8$ & $11.0 \pm 27.5$ & $9.2 \pm 5.3$ & $8.8 \pm 7.5$ & $10.6 \pm 19.3$ & 0.484 \\
\hline Hemoglobin (g/L) & $106.9 \pm 19.6$ & $112.3 \pm 18.8$ & $115.2 \pm 23.3$ & $118.1 \pm 22.9$ & $115.9 \pm 23.1$ & $113.1 \pm 25.8$ & $<0.001$ \\
\hline Albumin (g/L) & $32.3 \pm 7.0$ & $34.5 \pm 6.4$ & $36.1 \pm 6.0$ & $36.8 \pm 6.0$ & $36.5 \pm 6.1$ & $35.8 \pm 6.5$ & $<0.001$ \\
\hline Glucose (mmol/L) & $8.1 \pm 4.8$ & $8.0 \pm 3.8$ & $8.3 \pm 4.4$ & $7.4 \pm 3.2$ & $7.8 \pm 3.4$ & $8.5 \pm 5.3$ & 0.032 \\
\hline Bilirubin, total $(\mu \mathrm{mol} / \mathrm{L})$ & $44.2 \pm 104.8$ & $25.1 \pm 59.5$ & $25.2 \pm 60.1$ & $19.1 \pm 46.5$ & $16.2 \pm 30.3$ & $14.8 \pm 28.9$ & $<0.001$ \\
\hline Cholesterol, total (mmol/L) & $3.9 \pm 2.6$ & $3.8 \pm 1.3$ & $4.1 \pm 1.4$ & $4.1 \pm 1.3$ & $4.2 \pm 1.2$ & $4.4 \pm 1.4$ & $<0.001$ \\
\hline $\mathrm{Na}(\mathrm{mmol} / \mathrm{L})$ & $136.5 \pm 6.5$ & $137.3 \pm 5.2$ & $137.0 \pm 4.6$ & $138.0 \pm 4.8$ & $137.9 \pm 4.2$ & $137.7 \pm 4.5$ & 0.011 \\
\hline $\mathrm{K}(\mathrm{mmol} / \mathrm{L})$ & $4.2 \pm 0.8$ & $4.2 \pm 0.6$ & $4.2 \pm 0.6$ & $4.2 \pm 0.6$ & $4.2 \pm 0.6$ & $4.4 \pm 0.8$ & 0.018 \\
\hline Total $\mathrm{CO}_{2}(\mathrm{mmol} / \mathrm{L})$ & $22.4 \pm 4.9$ & $23.3 \pm 3.6$ & $23.1 \pm 3.9$ & $23.3 \pm 3.6$ & $22.7 \pm 3.8$ & $22.1 \pm 3.5$ & $<0.001$ \\
\hline Operation & $19(22.4)$ & 65 (29.3) & $128(32.0)$ & $120(30.7)$ & $61(24.1)$ & $74(28.4)$ & 0.212 \\
\hline Sepsis & $13(15.3)$ & $23(10.4)$ & $41(10.3)$ & $28(7.2)$ & $17(6.7)$ & $11(4.2)$ & 0.007 \\
\hline
\end{tabular}

Data are presented as number (\%) or mean \pm standard deviation.

AKI, acute kidney injury; RAAS, renin-angiotensin-aldosterone system; SBP, systolic blood pressure; WBC, white blood cell. 


\section{Results}

\section{Study population}

Among the 1,612 patients, the mean age, Charlson's comorbidity index value, and serum $\mathrm{Cr}$ levels at baseline were $65.8 \pm 16.0$ years, $2.8 \pm 2.2$, and $1.00 \pm 1.09 \mathrm{mg} / \mathrm{dL}$, respectively. The average SBP/diastolic BP (DBP) within 48 hours after admission and within 48 hours after AKI were 125.8/69.9 $\mathrm{mmHg}$ and 123.8/68.3 $\mathrm{mmHg}$, respectively. Of the 1,612 patients, $55.4 \%$ were males, $30.1 \%$ had hypertension, and $35.6 \%$ had diabetes mellitus. The median duration of AKI development was 1.0 (interquartile range: $0-4.0$ ) days after hospital admission. Additionally, the median duration of hospital stay and mean duration of total follow-up were 9.0 (interquartile range: 5-16) and 76.7 days, respectively. In total, 467 patients (29.0\%) underwent an operation and 133 patients $(8.3 \%)$ suffered from bacteremia episodes during admission (Table 1 ).

Table 2 shows the demographic and baseline characteristics according to SBP categories within 48 hours after AKI. Patients with low SBP were younger and leaner; less likely to have previous hypertension; had high levels of total bilirubin but low levels of baseline $\mathrm{Cr}$, hemoglobin, albumin, cholesterol, and total $\mathrm{CO}_{2}$; and had a high incidence of bacteremia.

\section{Outcomes}

Among the 1,612 patients, 90-day mortality rate, severe

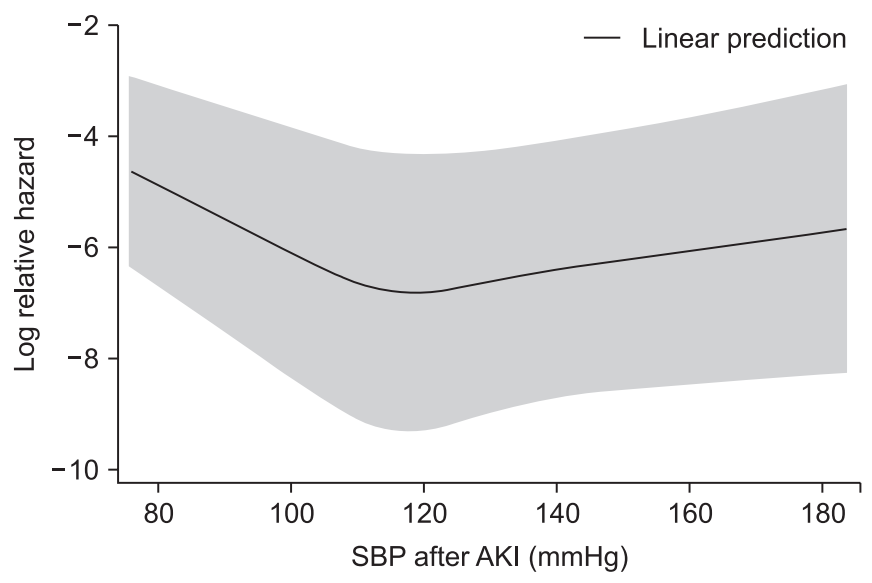

Figure 2. The relationship between average systolic blood pressure (SBP) within 48 hours after acute kidney injury (AKI) and composite outcome through a restricted cubic spline curve. The range area indicates 95\% confidence intervals.
AKI rate, and composite outcome rate for AKI inpatients were $11.5 \%(\mathrm{n}=185), 10.1 \%(\mathrm{n}=163)$, and $18.7 \%(\mathrm{n}=$ 302), respectively.

In the composite outcome group, a higher proportion of patients had diabetes mellitus and cancer as compared with in the group without the composite outcome. Additionally, the composite outcome group was older, leaner; had a higher white blood cell count and maximal $\mathrm{Cr}$ level during admission but low levels of albumin, sodium, total $\mathrm{CO}_{2}$, and hemoglobin; and had a high incidence of bacteremia and low incidence of operation. The relationship between SBP and composite outcome followed a Ushaped curve, with an increased event rate at both low and high SBP values (Fig. 2, 3). The average SBP after AKI predicted the composite outcome after adjusting for baseline variables (reference SBP: $120-129 \mathrm{mmHg}$; < 100 mmHg: HR 1.84, $P=0.015$; 100-109 mmHg: HR $1.56, P=0.038$; $110-119$ mmHg: HR $1.15, P=0.483 ; 130-$ 139 mmHg: HR 1.51, $P=0.045$; $\geq 140$ mmHg: HR 1.73, $P$ $=0.005$ ). The curve was relatively flat for SBPs of 110 to $130 \mathrm{mmHg}$ after AKI (Table 3). Separately, bias-corrected confidence intervals using bootstrap analysis $(n=10,000)$ to validate the SBP groups after AKI in predicting composite outcomes were calculated (reference SBP: 120-

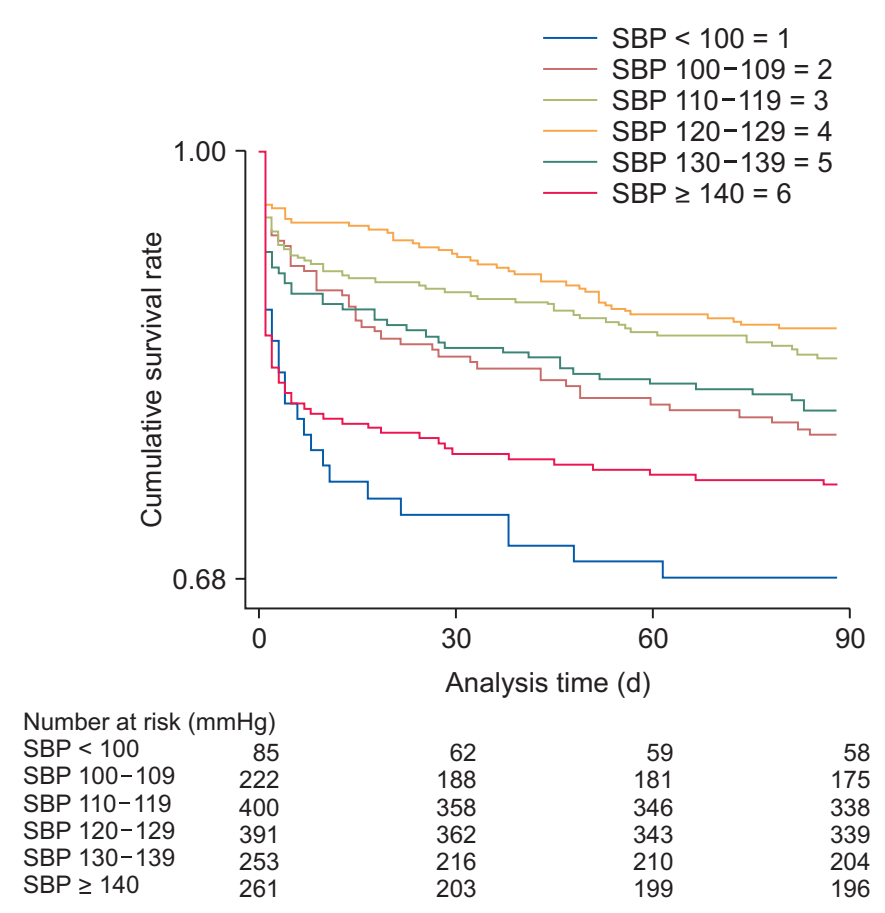

Figure 3. Kaplan-Meier curves for composite outcome of acute kidney injury patients during the 90-day period according to systolic blood pressure (SBP) within 48 hours after AKI. 
Baek, et al. Optimal systolic BP in AKI

Table 3. Adjusted hazard ratios for composite outcome according to average SBP

\begin{tabular}{|c|c|c|c|c|}
\hline Cox proportional hazards model & Composite outcome rate (n/total n, \%) & $\mathrm{HR}$ & $95 \% \mathrm{Cl}$ & $P$ value \\
\hline \multicolumn{5}{|c|}{ SBP within 48 hours after the development of AKI ( $\mathrm{mmHg}$ ) } \\
\hline$<100$ & $27 / 85(31.8)$ & 1.843 & $1.124-3.025$ & 0.015 \\
\hline $100-109$ & $47 / 222(21.2)$ & 1.557 & $1.025-2.363$ & 0.038 \\
\hline $110-119$ & $62 / 400(15.5)$ & 1.151 & $0.777-1.705$ & 0.483 \\
\hline $120-129$ & $52 / 391(13.3)$ & 1 & Reference & \\
\hline $130-139$ & 49/253 (19.4) & 1.514 & $1.008-2.274$ & 0.045 \\
\hline$\geq 140$ & $65 / 261(24.9)$ & 1.734 & $1.177-2.555$ & 0.005 \\
\hline
\end{tabular}

Multivariable, adjusted for age, gender, body mass index, Charlson's comorbidity index, hypertension, operation, bacteremia, white blood cell count, hemoglobin, albumin, cholesterol, alanine aminotransferase, sodium, total $\mathrm{CO}_{2}$, and maximal creatinine during admission.

AKI, acute kidney injury; $\mathrm{Cl}$, confidence interval; HR, hazard ratio; SBP, systolic blood pressure.

$129 \mathrm{mmHg} ;<100 \mathrm{mmHg}, 1.047-3.246 ; 100-109 \mathrm{mmHg}$, $1.014-2.391 ; 110-119 \mathrm{mmHg}, 0.770-1.721 ; 130-139$ $\mathrm{mmHg}, 0.972-2.360$; and $\geq 140 \mathrm{mmHg}, 1.063-2.829$ ).

\section{Subgroup analysis}

We performed a subgroup analysis according to the underlying disease to evaluate the effects of BP categories on the composite outcome in patients with AKI. Supplementary Tables 1 and 2 (available online) display the relation between $\mathrm{BP}$ and composite outcomes across the patient comorbidities. In 485 hypertensive patients, the proportion taking antihypertensive medication is shown in Table 1. In these patients, the risk of composite outcome increased 2.5-fold in the group with SBPs of less than $100 \mathrm{mmHg}$ as compared with the reference group (SBP of 120-129 $\mathrm{mmHg}$ ). In contrast, the relationship between SBP and composite outcome significantly followed a J-shaped curve, with an increased event rate observed at high SBP values in the nonhypertensive patients (reference SBP: 120-129 mmHg; 130-139 mmHg: HR 2.125; 95\% CI, 1.301-3.469; $\geq 140$ mmHg: HR 2.485; 95\% CI, 1.489-4.147).

Diabetic patients showed a similar pattern of BP and composite outcome as that of nonhypertensive patients. In nondiabetic patients, the risk of composite outcome increased 2.1-fold in the group with SBPs of less than $100 \mathrm{mmHg}$ in comparison with the reference group (SBP 120-130 $\mathrm{mmHg}$ ). A difference in the relationship between SBP and the composite outcome was observed, depending on the underlying disease.

\section{Discussion}

The main finding of this study was that the average SBP within 48 hours after AKI was a predictor for the composite outcome in noncritically ill hospitalized patients. After AKI, a U-shaped curve association was observed between the average SBP within $48 \mathrm{~h}$ after AKI and composite outcome, with the lowest event rates noted in the SBP range of approximately 110 to $129 \mathrm{mmHg}$.

The ideal target BP has been controversial in the general management of hypertension. Prior to the publication of SPRINT, the international guidelines for hypertension recommended a BP of below 140/90 mmHg was optimal in most patients with hypertension [21-23]. However, as a more recent randomized controlled trial, SPRINT showed that an SBP of less than $120 \mathrm{mmHg}$ as compared with of less than $140 \mathrm{mmHg}$ in patients with hypertension but without diabetes resulted in lower rates of fatal and nonfatal major cardiovascular events and death [4]. Therefore, a recent international guideline reported that a BP target of less than $130 / 80 \mathrm{mmHg}$ is indicated [24,25].

Meanwhile, several studies have reported that too low of a BP may be harmful in patients with AKI as in those with acute illnesses such as acute coronary syndrome and stroke [5-8,10-12,16,17]. Therefore, in patients with septic AKI or hepatorenal syndrome, a target MAP of 65 to $70 \mathrm{mmHg}$ is suggested. A higher target MAP of 80 to 85 $\mathrm{mmHg}$ is also suggested in patients with chronic hypertension [17]. However, most studies on hemodynamic targets in patients with AKI have been conducted in critically ill patients [9-16]. Although Liu et al [26] reported that relative decreases in SBP and DBP and MAP were associated with the development of AKI and a decrease in SBP is a significant independent predictor of the devel- 
opment of severe AKI in noncritically ill patients, there is limited knowledge concerning the optimal BP in general ward patients with AKI. To our knowledge, this is the first study to investigate the hemodynamic target in noncritically ill ward patients with AKI relative to severe AKI or mortality. As this study was based on general ward hospitalization, with the exception of high-risk AKI in critically ill patients, it could give physicians realistic guidance for treating hospitalized patients. Furthermore, internal validation was performed using bootstrap analysis, and the prediction models used might be robust.

Our data showed that a U-shaped curve association existed between the average SBP within 48 hours after AKI and a composite outcome, with the lowest event rates present in the SBP range of approximately 110 to 129 $\mathrm{mmHg}$ in these patients after the development of AKI. Importantly, however, the present study does not prove a causal relationship between low/high BP and clinical outcomes because of its retrospective nature. Instead, a U-shaped curve association between the average SBP after AKI and the composite outcome can be inferred as follows: In accordance with previous studies $[10,11]$, too low of a BP can be associated with poor prognosis because BP has been thought to be essential for organ perfusion [3]. We thought that normotensive ischemic AKI may be a cause of severe AKI in patients with normalrange or high $\mathrm{BP}$ after AKI $[26,27]$.

MAP has been widely used as an index for optimal BP and, recently, mean perfusion pressure calculated together with central venous pressure was introduced as a more useful indicator in the context of preventing AKI $[3,28]$. Our data showed that only SBP was an independent predictor of the clinical outcome; DBP and MAP were not predictors (data not shown). A previous study of noncritically ill ward patients suggested that only SBP is an independent predictor of the development of severe AKI [26], which is consistent with our data. Moreover, noncritically ill ward patients cannot measure the mean perfusion pressure or central venous pressure, which is known to better reflect renal perfusion than MAP does.

We further analyzed the association between SBP and clinical outcomes, adjusting antihypertensive medications to the existing variables in 485 hypertensive patients. The HR showed a similar pattern, but there was no correlation between SBP and the composite outcome (data not shown). The reason for losing statistical signifi- cance is presumed to be that the multivariate analysis included too many variables as compared with the 485 patients after adjusting antihypertensive agents to the existing variables. In terms of antihypertensive therapy, the use of renin-angiotensin receptor blockade or betablockers was associated with favorable outcomes (HR $0.44, P=0.001$ and HR $0.43, P=0.006$ ), while diuretics were associated with a poor prognosis (HR 1.74; $P=$ 0.021).

Our study has several limitations. First, it is a retrospective analysis of a heterogeneous population with different comorbidities admitted to a single tertiary hospital. Therefore, the results of our findings cannot be generalized. We also cannot prove a causal relationship between BP and the clinical outcomes. Therefore, we only mentioned the association between BP and the outcomes; further prospective research is needed to prove causality. Furthermore, we cannot determine the exact etiology of AKI and the causes of death because we did not have sufficient data. Fourth, with regard to defining AKI, we did not consider time (within 48 hours or 7 days) according to the guidelines proposed by the KDIGO [20]; however, the majority of AKI episodes occurred during the early period of hospitalization (within 7 days) [29]. Fifth, with regard to the definition of $\mathrm{AKI}$, if the Cr value measured at less than 180 days prior to admission was unavailable, the Cr level was estimated using the MDRD study equation adjusted for the Korean ethnic coefficient, assuming that the baseline eGFR was $75 \mathrm{~mL} / \mathrm{min} / 1.73 \mathrm{~m}^{2}$. Although this approach has been used in prior research [30-39] as well as studies of AKI epidemiology using the RIFLE (Risk, Injury, Failure, Loss of kidney function, and End-stage kidney disease) protocol and has recently been validated [40], this assumption was applied to only $25.6 \%$ of patients.

In conclusion, we performed a retrospective cohort study on noncritically ill patients who developed AKI and found that a U-shaped curve association existed between the average SBP within 48 hours after AKI and a composite outcome, with the lowest event rates found in the SBP range of approximately 110 to $129 \mathrm{mmHg}$.

\section{Conflicts of interest}

All authors have no conflicts of interest to declare. 


\section{Acknowledgments}

This research was supported by a grant from the Korea Health Technology R\&D Project through the Korea Health Industry Development Institute (KHIDI), funded by the Ministry of Health \& Welfare, Republic of Korea (grant no. HI17C1827).

\section{Authors' contributions}

Seon Ha Baek and Sejoong Kim participated in the study design, data collection, performed the statistical analysis, and wrote the manuscript. Ho Jun Chin provided intellectual content of critical importance to the work and technical support. Ki Young Na and Dong-Wan Chae participated in the coordination and helped to draft the manuscript. All authors read and approved the final manuscript.

\section{References}

[1] Section 1: introduction and methodology. Kidney Int Suppl (2011) 2012;2:13-18.

[2] Section 3: prevention and treatment of AKI. Kidney Int Suppl (2011) 2012;2:37-68.

[3] Sato R, Luthe SK, Nasu M. Blood pressure and acute kidney injury. Crit Care 2017;21:28.

[4] SPRINT Research Group, Wright JT Jr, Williamson JD, et al. A randomized trial of intensive versus standard bloodpressure control. N Engl J Med 2015;373:2103-2116.

[5] Bangalore S, Qin J, Sloan S, Murphy SA, Cannon CP; PROVE IT-TIMI 22 Trial Investigators. What is the optimal blood pressure in patients after acute coronary syndromes?: relationship of blood pressure and cardiovascular events in the PRavastatin OR atorVastatin Evaluation and Infection Therapy-Thrombolysis In Myocardial Infarction (PROVE ITTIMI) 22 trial. Circulation 2010;122:2142-2151.

[6] Messerli FH, Mancia G, Conti CR, et al. Dogma disputed: can aggressively lowering blood pressure in hypertensive patients with coronary artery disease be dangerous? Ann Intern Med 2006;144:884-893.

[7] Protogerou AD, Safar ME, Iaria P, et al. Diastolic blood pressure and mortality in the elderly with cardiovascular disease. Hypertension 2007;50:172-180.

[8] Wohlfahrt P, Krajcoviechova A, Jozifova M, et al. Low blood pressure during the acute period of ischemic stroke is as- sociated with decreased survival. J Hypertens 2015;33:339345.

[9] Davison DL, Patel K, Chawla LS. Hemodynamic monitoring in the critically ill: spanning the range of kidney function. Am J Kidney Dis 2012;59:715-723.

[10] Badin J, Boulain T, Ehrmann S, et al. Relation between mean arterial pressure and renal function in the early phase of shock: a prospective, explorative cohort study. Crit Care 2011;15:R135.

[11] Poukkanen M, Wilkman E, Vaara ST, et al; FINNAKI Study Group. Hemodynamic variables and progression of acute kidney injury in critically ill patients with severe sepsis: data from the prospective observational FINNAKI study. Crit Care 2013;17:R295.

[12] Thomson R, Meeran H, Valencia O, Al-Subaie N. Goaldirected therapy after cardiac surgery and the incidence of acute kidney injury. J Crit Care 2014;29:997-1000.

[13] Bourgoin A, Leone M, Delmas A, Garnier F, Albanèse J, Martin C. Increasing mean arterial pressure in patients with septic shock: effects on oxygen variables and renal function. Crit Care Med 2005;33:780-786.

[14] Legrand M, Dupuis C, Simon C, et al. Association between systemic hemodynamics and septic acute kidney injury in critically ill patients: a retrospective observational study. Crit Care 2013;17:R278.

[15] Asfar P, Meziani F, Hamel JF, et al; SEPSISPAM Investigators. High versus low blood-pressure target in patients with septic shock. N Engl J Med 2014;370:1583-1593.

[16] Raimundo M, Crichton S, Syed Y, et al. Low systemic oxygen delivery and BP and risk of progression of early AKI. Clin J Am Soc Nephrol 2015;10:1340-1349.

[17] Mohsenin V. Practical approach to detection and management of acute kidney injury in critically ill patient. J Intensive Care 2017;5:57.

[18] Levey AS, Stevens LA, Schmid CH, et al; CKD-EPI (Chronic Kidney Disease Epidemiology Collaboration). A new equation to estimate glomerular filtration rate. Ann Intern Med 2009;150:604-612.

[19] Lee CS, Cha RH, Lim YH, et al. Ethnic coefficients for glomerular filtration rate estimation by the Modification of Diet in Renal Disease study equations in the Korean population. J Korean Med Sci 2010;25:1616-1625.

[20] Section 2: AKI definition. Kidney Int Suppl (2011) 2012;2:1936.

[21] Tykarski A, Narkiewicz K, Gaciong Z, et al. 2015 guidelines for the management of hypertension. Recommendations of 
the Polish Society of Hypertension-short version. Kardiol Pol 2015;73:676-700.

[22] James PA, Oparil S, Carter BL, et al. 2014 evidence-based guideline for the management of high blood pressure in adults: report from the panel members appointed to the Eighth Joint National Committee (JNC 8). JAMA 2014;311:507-520.

[23] Weber MA, Schiffrin EL, White WB, et al. Clinical practice guidelines for the management of hypertension in the community: a statement by the American Society of Hypertension and the International Society of Hypertension. $J$ Clin Hypertens (Greenwich) 2014;16:14-26.

[24] Whelton PK, Carey RM, Aronow WS, et al. 2017 ACC/AHA/ AAPA/ABC/ACPM/AGS/APhA/ASH/ASPC/NMA/PCNA Guideline for the prevention, detection, evaluation, and management of high blood pressure in adults: executive summary: A Report of the American College of Cardiology/ American Heart Association Task Force on Clinical Practice Guidelines. J Am Coll Cardiol 2018;71:2199-2269.

[25] Brown MJ. Comments on the 2018 ESC/ESH Guidelines for the management of arterial hypertension. Hypertens News [Internet]. 2018 Jun 8 [cited 2019 Mar 18]. Available from: http://ish-world.com/data/uploads/June_8_Guidelines_ MJB.pdf.

[26] Liu YL, Prowle J, Licari E, Uchino S, Bellomo R. Changes in blood pressure before the development of nosocomial acute kidney injury. Nephrol Dial Transplant 2009;24:504511.

[27] Abuelo JG. Normotensive ischemic acute renal failure. $N$ Engl J Med 2007;357:797-805.

[28] Forni LG, Joannidis M. Blood pressure deficits in acute kidney injury: not all about the mean arterial pressure? Crit Care 2017;21:102.

[29] McGregor TL, Jones DP, Wang L, et al. Acute kidney injury incidence in noncritically ill hospitalized children, adolescents, and young adults: a retrospective observational study. Am J Kidney Dis 2016;67:384-390.

[30] Hoste EA, Clermont G, Kersten A, et al. RIFLE criteria for acute kidney injury are associated with hospital mortality in critically ill patients: a cohort analysis. Crit Care 2006;10:
R73.

[31] Uchino S, Bellomo R, Goldsmith D, Bates S, Ronco C. An assessment of the RIFLE criteria for acute renal failure in hospitalized patients. Crit Care Med 2006;34:1913-1917.

[32] Bagshaw SM, George C, Dinu I, Bellomo R. A multi-centre evaluation of the RIFLE criteria for early acute kidney injury in critically ill patients. Nephrol Dial Transplant 2008;23: 1203-1210.

[33] Ostermann M, Chang RW. Acute kidney injury in the intensive care unit according to RIFLE. Crit Care Med 2007;35: 1837-1843.

[34] Ali T, Khan I, Simpson W, et al. Incidence and outcomes in acute kidney injury: a comprehensive population-based study. J Am Soc Nephrol 2007;18:1292-1298.

[35] Bell M, Liljestam E, Granath F, Fryckstedt J, Ekbom A, Martling CR. Optimal follow-up time after continuous renal replacement therapy in actual renal failure patients stratified with the RIFLE criteria. Nephrol Dial Transplant 2005;20:354-360.

[36] Cruz DN, Bolgan I, Perazella MA, et al; North East Italian Prospective Hospital Renal Outcome Survey on Acute Kidney Injury (NEiPHROS-AKI) Investigators. North East Italian Prospective Hospital Renal Outcome Survey on Acute Kidney Injury (NEiPHROS-AKI): targeting the problem with the RIFLE Criteria. Clin J Am Soc Nephrol 2007;2:418-425.

[37] Perez-Valdivieso JR, Bes-Rastrollo M, Monedero P, de Irala J, Lavilla FJ. Prognosis and serum creatinine levels in acute renal failure at the time of nephrology consultation: an observational cohort study. BMC Nephrol 2007;8:14.

[38] Coca SG, Bauling P, Schifftner T, Howard CS, Teitelbaum I, Parikh CR. Contribution of acute kidney injury toward morbidity and mortality in burns: a contemporary analysis. Am J Kidney Dis 2007;49:517-523.

[39] Maccariello E, Soares M, Valente C, et al. RIFLE classification in patients with acute kidney injury in need of renal replacement therapy. Intensive Care Med 2007;33:597-605.

[40] Závada J, Hoste E, Cartin-Ceba R, et al; AKI6 investigators. A comparison of three methods to estimate baseline creatinine for RIFLE classification. Nephrol Dial Transplant 2010;25:3911-3918. 